\title{
OPEN
}

\section{Author Correction: Genome-wide identification of and functional insights into the late embryogenesis abundant (LEA) gene family in bread wheat (Triticum aestivum)}

\author{
Hao Liu, Mingyan Xing, Wenbo Yang, Xiaoqian Mu, Xin Wang, Feng Lu, Yao Wang \& \\ Linsheng Zhang
}

Correction to: Scientific Reports https://doi.org/10.1038/s41598-019-49759-w, published online 16 September 2019

This Article contains errors.

In the Results section, the subheading,

"Enhancement of the tolerance of recombinant E. coli and yeast cells to salt and heat-".

should read:

"Enhancement of the tolerance of recombinant E. coli and yeast cells to salt and heat-stress".

Furthermore, in the Results section, under the subheading 'Determining the endogenous wheat ABA content and expression patterns of TaLEA genes under ABA and abiotic stress treatments',

"To confirm the results of the microarray data, we selected 29 TaLEA genes belonging to LEA_1, LEA_2, LEA_3, LEA_4, LEA_5, LEA_6, SMP and three DHN subfamilies to investigate the expression patterns of TaLEA genes in wheat seedlings subjected to ABA and abiotic stress treatments by real-time PCR (Fig. 5).”

should read:

"To confirm the results of the microarray data, we selected 22 TaLEA genes belonging to LEA_1, LEA_2, LEA_4, LEA_5, SMP and three DHN subfamilies to investigate the expression patterns of TaLEA genes in wheat seedlings subjected to ABA and abiotic stress treatments by real-time PCR (Fig. 5).'

In the Methods section, under the subheading 'In vivo assay of the stress tolerance of transformed E. coli,

"Heat and salt tolerance assays were performed as described previously"."

should read:

"Heat and salt tolerance assays were performed as described previously ${ }^{26 "}$

Finally, this Article contains errors in Figure 5 and Supplementary Table S2, where the primers of TaLEA_35, TaLEA_6-1, TaLEA_6-2, TaLEA_6-3, TaSMP8 and TaSMP9 are incorrectly included. In addition, Figure 5 incorrectly includes the primer of TaLEA_3-3, and Supplementary Table S2 incorrectly includes the primer of TaLEA_3-4. The correct Figure 5 and Supplementary Table S2 appear below as Figure 1 and Table 1 respectively. 


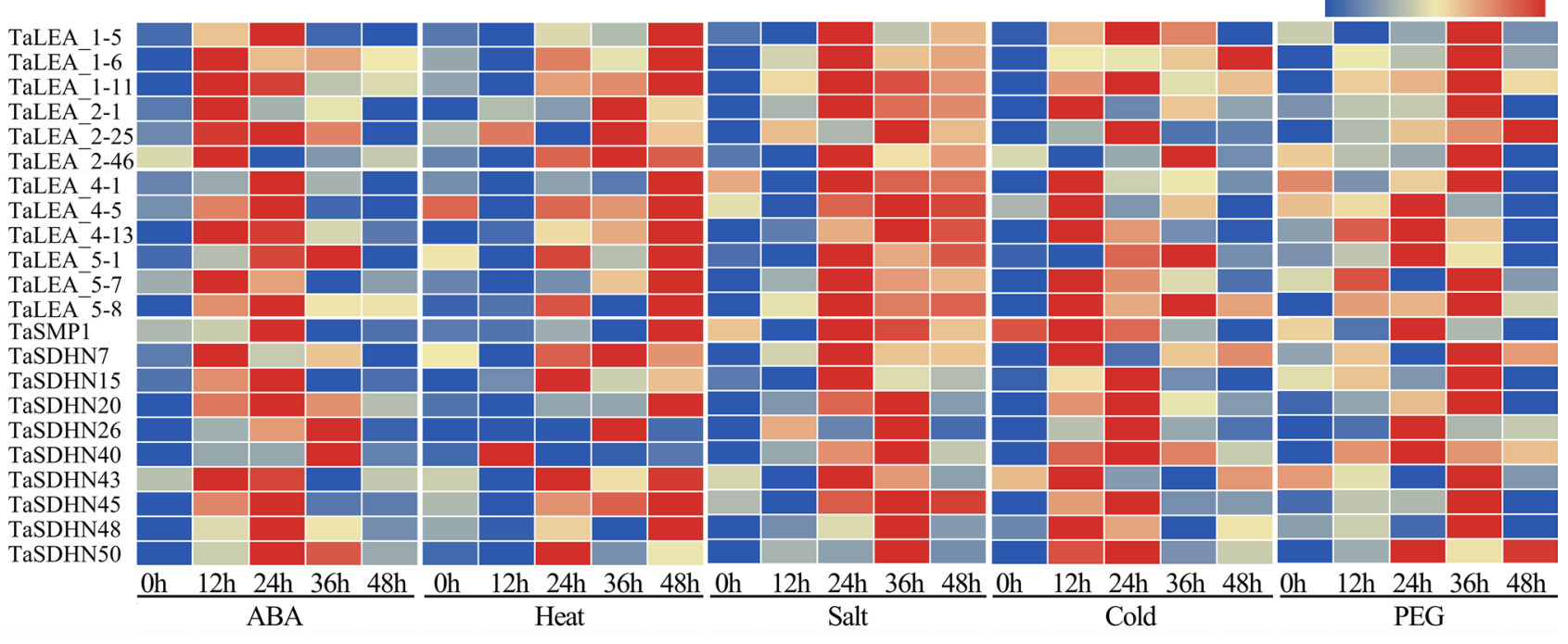

Figure 1. Expression of TaLEA genes in response to ABA, heat, $\mathrm{NaCl}$, cold, and PEG treatments determined by real-time PCR. The expression level of wheat actin was used as the internal control to standardize the RNA samples for each reaction. The values are the mean \pm SE from three samples. 


\begin{tabular}{|c|c|c|}
\hline Genes & Primer name & Sequence $\left(5^{\prime}-3^{\prime}\right)$ \\
\hline \multirow{2}{*}{ Actin } & Qactin F & ACCCAACCAGAAACAGCAAC \\
\hline & Qactin R & CTCATCCAACGAAACGGAAT \\
\hline \multirow{2}{*}{ TaLEA_1-5 } & QTaLEA_1-5 F & AAGATGAAGGACGGCGCG \\
\hline & QTaLEA_1-5 R & CTTGTGGATGTGGTGCGAC \\
\hline \multirow{2}{*}{ TaLEA_1-6 } & QTaLEA_1-6 F & TGTGCATCGATTCCTTCACG \\
\hline & QTaLEA_1-6 R & GCATGCATGACTCATCGCTT \\
\hline \multirow{2}{*}{ TaLEA_1-11 } & QTaLEA_1-11 F & GAAGGCCAAGACCAAGATCG \\
\hline & QTaLEA_1-11 R & CTTGTGGATGCGGTGCTC \\
\hline \multirow{2}{*}{ TaLEA_2-1 } & QTaLEA_2-1 F & CTCСТTCAACCTCAAGTGCG \\
\hline & QTaLEA_2-1 R & GATGAGGAAGTCGTAGGGCA \\
\hline \multirow{2}{*}{ TaLEA_2-25 } & QTaLEA_2-25 F & ACGGAGAGGATGATGAGCAG \\
\hline & QTaLEA_2-25 R & CGTCGCTCTTGAACGTGTAG \\
\hline \multirow{2}{*}{ TaLEA_2-46 } & QTaLEA_2-46F & GTGAGCTTCAAGGGCATGAC \\
\hline & QTaLEA_2-46 R & GATGAGGAAGTCGTAGGGCA \\
\hline \multirow{2}{*}{ TaLEA_4-1 } & QTaLEA_4-1 F & AGTACACCAAGGACTCCGC \\
\hline & QTaLEA_4-1 R & TTTGTGATGGTCTCGGTGGT \\
\hline \multirow{2}{*}{ TaLEA_4-5 } & QTaLEA_4-5 F & ACACGCAGGACAGGACATAC \\
\hline & QTaLEA_4-5 R & GTGATCCTCTCGGTGGTGTC \\
\hline \multirow{2}{*}{ TaLEA_4-13 } & QTaLEA_4-13 F & GAGAAGGCTGGACAGGTGAT \\
\hline & QTaLEA_4-13 R & GGTCTCGTTGGTCTTGTCCT \\
\hline \multirow{2}{*}{ TaLEA_5-1 } & QTaLEA_5-1 F & GGAGGAGGGGTACAGTGAGA \\
\hline & QTaLEA_5-1 R & CTCGTCGATGTCTATGCCCT \\
\hline \multirow{2}{*}{ TaLEA_5-7 } & QTaLEA_5-7 F & AGGAGAAACTCGCTGAAGGG \\
\hline & QTaLEA_5-7 R & TGGTCTTGAACTTGGACTCGT \\
\hline \multirow{2}{*}{ TaLEA_5-8 } & QTaLEA_5-8 F & CTACGAGGCGCAGGAGAAA \\
\hline & QTaLEA_5-8 R & GACTCATCGTTGGTGCTCAG \\
\hline \multirow{2}{*}{ TaSMP1 } & QTaSMP1 F & GTCAATATCCCTGGTGGCCT \\
\hline & QTaSMP1 R & CTTGTCGTCCTTGTTTCGGG \\
\hline \multirow{2}{*}{ TaDHN7 } & QTaDHN7 F & GTAGCCGAGCAAAAGACTCG \\
\hline & QTaDHN7 R & CCGTGGTCTGCTCCTTGTTA \\
\hline \multirow{2}{*}{ TaDHN15 } & QTaDHN15F & GGCAAAGATGGAGTTCCAAG \\
\hline & QTaDHN15 R & CAGTATCCCACCGGTCTTGT \\
\hline \multirow{2}{*}{ TaDHN20 } & QTaDHN20 F & GGAGTACCAGGGACATCAGC \\
\hline & QTaDHN20 R & CATCCTCAGACGAGCTGGA \\
\hline \multirow{2}{*}{ TaDHN26 } & QTaDHN26F & GCGGCAGCTACTTGAGAGTT \\
\hline & QTaDHN26 R & AACGTCCCGGGTACATACAA \\
\hline \multirow{2}{*}{ TaDHN40 } & QTaDHN40 F & AGAAGGGCATCATGGAGAAC \\
\hline & QTaDHN40 R & GTCATTCCAGTGTGTCCCTG \\
\hline \multirow{2}{*}{ TaDHN43 } & QTaDHN43 F & AGTTACCGGCGAGAACATCA \\
\hline & QTaDHN43 R & GACTTCCCGTAGTTGCCATC \\
\hline \multirow{2}{*}{ TaDHN45 } & QTaDHN45 F & CAGCATGGACACACTGGAAT \\
\hline & QTaDHN45 R & GCAGCTTCTCCTTCACCTTG \\
\hline \multirow{2}{*}{ TaDHN48 } & QTaDHN48F & CAGTCACAAAGCCAAAGCAA \\
\hline & QTaDHN48 R & GACCAGCTCCTCCTCCTTCT \\
\hline \multirow{2}{*}{ TaDHN50 } & QTaDHN50F & GAGCCCGAGGTTAAGAAGGA \\
\hline & QTaDHN50 R & TGATCACCTCACCGTTGTCA \\
\hline
\end{tabular}

Table 1. Real time PCR primers of TaLEA genes. 
(c) (i) Open Access This article is licensed under a Creative Commons Attribution 4.0 International cc) License, which permits use, sharing, adaptation, distribution and reproduction in any medium or format, as long as you give appropriate credit to the original author(s) and the source, provide a link to the Creative Commons license, and indicate if changes were made. The images or other third party material in this article are included in the article's Creative Commons license, unless indicated otherwise in a credit line to the material. If material is not included in the article's Creative Commons license and your intended use is not permitted by statutory regulation or exceeds the permitted use, you will need to obtain permission directly from the copyright holder. To view a copy of this license, visit http://creativecommons.org/licenses/by/4.0/.

(C) The Author(s) 2020 